\title{
Electrostatic forces in fibrous filters-a review
}

\author{
Chiu-Sen Wang * \\ Department of Public Health, National Taiwan University, Taipei, Taiwan
}

Dedicated to the late Professor Koichi Iinoya

\begin{abstract}
Among various devices for removing submicrometer particles from a gas stream, fibrous filters have the advantages of providing high collection efficiencies at low-pressure drop. Even in the absence of electrostatic forces, a fibrous filter captures particles efficiently by inertial impaction, interception, and convective Brownian diffusion. Application of electrostatic forces can significantly increase the efficiency of collection. The process of aerosol filtration in the presence of electrostatic forces is complicated. Although past studies have led to a good understanding of the filtration process in clean filters, there is a marked gap between theory and experiment of the change in collection efficiency with particle loading in a filter in the presence of electrostatic forces. This paper gives a review on both theoretical and experimental studies on the application of electrostatic forces in filtration and discusses the needs for further studies on the effect of particle loading. (C) 2001 Elsevier Science B.V. All rights reserved.
\end{abstract}

Keywords: Fibrous filters; Electrostatic forces; Collection efficiency; Particle loading

\section{Introduction}

Fibrous filters are efficient and low-pressure drop devices for capturing submicrometer particles, which are difficult to remove from a gas stream by other devices such as cyclones. Consequently, fibrous filters have found applications in disposable respirators, automotive cabin air filters, vacuum cleaner bags, indoor air filters, and industrial gas cleaning devices. A fibrous filter consists of loosely packed fibers with a preferred orientation across the direction of gas flow. The ratio of the total volume of all the fibers in a filter to the volume of the filter, termed the packing density, is in the range of $1-15 \%$. The lowpressure drop across a fibrous filter is a result of low packing density. Materials suitable for use as filter media include glass, polypropylene, polycarbonate, ceramic, and stainless steel, some of which can be used in high-temperature filtration. The fibers in a filter generally have a circular or rectangular cross-section, with a narrow diameter (or width) distribution. The fiber diameter in most filters is smaller than $100 \mu \mathrm{m}$ and can be as small as 0.01 $\mu \mathrm{m}$. The velocity of gas flow at the surface of a filter, termed the face velocity, is in the order of $0.1 \mathrm{~m} / \mathrm{s}$.

As a particle-laden gas stream approaches a fiber, particles suspended in the stream move towards the fiber

\footnotetext{
* Tel.: +886-2-2341-0065; fax: +886-2-2351-6701.

E-mail address: cswang@ccms.ntu.edu.tw (C.-S. Wang).
}

surface by a number of forces. In the absence of electrostatic forces, a small particle departs from the gas streamline it originally follows, mainly by thermal forces and particle inertia. When a particle comes within one particle radius of the surface of a fiber, it makes contact with the fiber and deposits (such a deposition mechanism, termed interception, is an effect of the finite size of a particle). The relative contributions of thermal forces and particle inertia to deposition are functions mainly of particle diameter, gas velocity, and fiber diameter. In general, particle inertia makes a greater contribution for particles larger than $1 \mu \mathrm{m}$, while Brownian motion caused by thermal forces plays a greater role for particles smaller than 0.1 $\mu \mathrm{m}$. For particles in the size range of $0.05-0.5 \mu \mathrm{m}$, both particle inertia and thermal forces are relatively weak. As a consequence, the collection efficiency of a fibrous filter has a minimum in this size range. To design a filter involves the selection of fiber material, fiber diameter, packing density, filter thickness, and face velocity, with the objective of achieving a specified collection efficiency for given particle size, gas flow rate, pressure drop across the filter, temperature, relative humidity, and pressure.

Application of electrostatic forces can significantly augment the collection efficiency of a fibrous filter. This is particularly useful for improving the collection of particles in the size range of $0.15-0.5 \mu \mathrm{m}$, which are difficult to capture by other mechanisms. With the help of electrostatic forces, a filter can achieve a specified collection 
efficiency at a lower packing density and thereby a lower resistance to gas flow in the filter. The variables that influence the collection efficiency of a filter in the presence of electrostatic forces include chemical compositions of particles and fibers, charges on particles, surface charge density of fibers, and the intensity of the externally applied electric field, in addition to those variables that affect the collection efficiency in the absence of electrostatic forces.

An important aspect in filtration is the accumulation of particles in the filter and the effects of particle loading on collection efficiency and pressure drop across the filter. The effects of particle loading differ between solid particles and liquid droplets.

This paper gives a review of the theories and experiments on fibrous filtration in the presence of electrostatic forces and discusses the needs for further studies on the effect of particle accumulation.

\section{Electrostatic forces used in fibrous filters}

Electrostatic effects are generally present in filtration processes because particles and fibers are charged to some extent. However, the effects are insignificant unless the particles or fibers are highly charged. There are basically two ways of applying significant electrostatic forces to augment the collection efficiency of a filter: (1) charging the airborne particles and (2) creating an electric field in the filter. A charged particle polarizes the fiber and, as a result, experiences an image force that is equal to the Coulombic force between the charge on the particle and an equal, but opposite, charge placed inside the fiber at a position corresponding to the optical image of the particle. The image force is not very important, unless the particle carries very high charges.

An externally applied electric field polarizes the fiber as well as the particles. The polarized fiber acts as a linear dipole and creates a nonuniform field. The charge on a particle in such a nonuniform electric field experiences a force, in addition to the force exerted by the externally applied field. The nonuniform electric field also exerts a force on the induced dipole in the particle, but the force is unimportant for small particles since it is proportional to the particle volume.

Charging the fibers can also create an electric field in a filter. Examples of filters with charged fibers include the

Table 1

The expressions for various electrostatic forces acting on a particle in the vicinity of a fiber

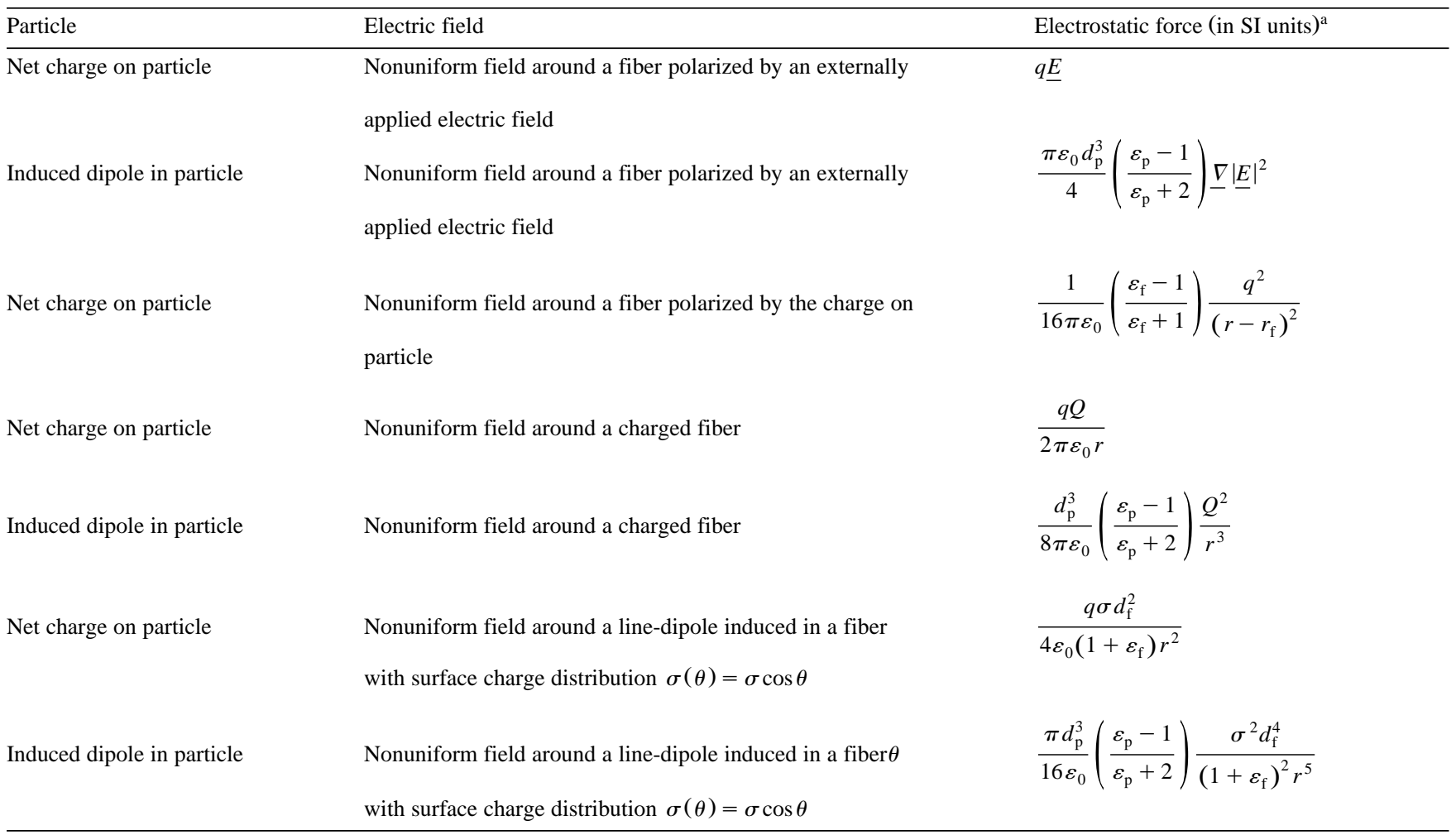

${ }^{\mathrm{a}} \varepsilon_{0}=$ permittivity of free space; $\varepsilon_{\mathrm{p}}=$ dielectric constant of particle; $\varepsilon_{\mathrm{f}}=$ dielectric constant of fiber; $d_{\mathrm{p}}=$ particle diameter; $d_{\mathrm{f}}=$ fiber diameter; $r_{\mathrm{f}}=$ fiber radius; $q=$ net charge on particle; $Q=$ net charge on unit length of fiber; $E_{0}=$ externally applied electric field; $\sigma=$ surface charge density; $r=$ radial distance from fiber axis; $\theta=$ polar angular coordinate; $\underline{E}=$ electric field with components: $E_{\mathrm{r}}=\left[1+\left(\frac{\varepsilon_{\mathrm{f}}-1}{\varepsilon_{\mathrm{f}}+1}\right) \frac{r_{\mathrm{f}}^{2}}{r^{2}}\right] E_{0} \cos \theta, \quad E_{\theta}=\left[1+\left(\frac{\varepsilon_{\mathrm{f}}-1}{\varepsilon_{\mathrm{f}}+1}\right) \frac{r_{\mathrm{f}}^{2}}{r^{2}}\right] E_{0} \sin \theta$. 
Hansen filter (see Ref. [1]) and some types of electret filters (see Ref. [2]). The Hansen filter was the first significant application of electrostatic forces in aerosol filtration. Hansen found that the collection efficiency increased markedly when a wool filter pad was powdered with colophony resin particles. The improvement results from the action of electrostatic forces. Resin particles, about $1 \mu \mathrm{m}$ in diameter, are negatively charged by contact (triboelectric charging). Because resin is a good electrical insulator, the charge remains on a resin particle for a long time. Electret filters are made of dielectric fibers that have a quasi-permanent electrical charge. Manufacturing processes of electret filters include corona charging, triboelectric charging, and induction charging [3].

A charged fiber creates in its vicinity an electric field that exerts a force on a charged particle. The field created by a charged fiber can also polarize a particle, but the force on a polarized particle is unimportant for small particles. Some types of electret fibers carry a permanent line dipole charge [4] and therefore create a nonuniform field. The charge on a particle experiences a force in such a field. The field also induces a dipole charge in a particle, but it is not important for small particles.

Table 1 summarizes the expressions for various electrostatic forces acting on a particle in the vicinity of a fiber.

\section{Initial stage of filtration}

Earlier studies, both experimental and theoretical, focused on the collection efficiency and pressure drop of clean filters. Davies [1] reviewed the studies carried out before early 1970s. The reviews given by Pich [5], Brown [3], and Wang [6] cover more recent developments.

Experiments have verified that electrostatic forces can greatly enhance the collection efficiency of a fibrous filter. When the fibers are clean, particles deposit directly on the surface of fibers. In principle, it is possible to calculate the rate at which particles deposit on a fiber from the equation of motion, taking into account all the forces acting on the particles. Because the boundary conditions for the flow field around a fiber in a real filter are complicated, most theoretical analyses approximate a filter by a fiber-array representation consisting of a number of cylinders arranged in a certain regular manner. The model of staggered fibers shown in Fig. 1 has proved to be a good representation. It is one of the simplest models that has taken into account the depth of a fibrous filter. Approximating the hexagonal cell shown in Fig. 1 by a circular cell, Kuwabara [7] obtained a solution to the problem of viscous flow around a fiber at low Reynolds number.

Earlier studies on the theory of particle capture in the presence of electrostatic forces have focused on uniformly charged fibers or on fibers placed in an externally applied electric field [8,9]. More recently, Wu et al. [10] gave theoretical expressions for single-fiber efficiencies in a

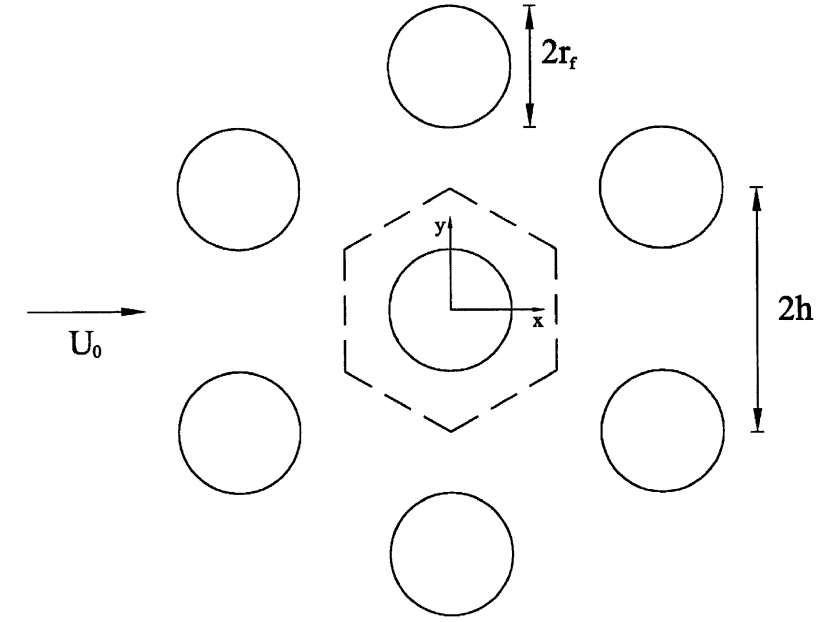

Fig. 1. A model of staggered fibers. Each fiber has a radius $r_{\mathrm{f}}$ and two neighboring fibers are separated by a distance $2 h$. The approaching air flow has a face velocity $U_{0}$.

uniform electric field, taking into account the effect of interception. For fibers carrying a permanent dipole moment, Brown [11] derived the expressions of capture efficiency for both charged and neutral particles. Brown [12] also developed a theory which shows that charged fibers parallel to the airflow are more efficient than charged fibers perpendicular to the airflow for capturing charged particles. Subsequently, Banks and Kurowski [13] made a theoretical study on the electrical enhancement of filters with randomly oriented fibers.

Theoretical predications are in general agreement with experimental results obtained in recent years. Using the Kuwabara flow, Lathrache et al. [14] calculated the collection efficiency of charged fibers for charged particles. Their calculations are in good agreement with the data reported by Baumgartner and Loffler [15]. Pich et al. [16] derived a solution for the capture of unipolarly charged particles by bipolarly charged fibers, which is also in good agreement with experimental data. Baumgartner et al. [17] developed an experimental method for recording the motion of particles during the process of deposition onto electret fibers. The recorded particle trajectories and the results of numerical simulations are in good agreement. More recently, Romay et al. [18] obtained empirical power law expressions for single-fiber efficiencies in commercial electret filters which were in good agreement with those predicted by theory [11].

\section{Particle loading}

The manner in which particles accumulate in a fibrous filter differs between solid particles and liquid droplets. Deposited liquid droplets tend to form a thin film on the fiber surface. Experiments showed that deposits of solid particles have a tendency to take the shape of irregular 
chains and bend in the absence of electrostatic forces [19], but the dendrites appear quite straight when electric fields are present [20].

An interesting aspect of aerosol filtration is that fibers with a diameter closer to that of particles are better collectors than fibers of larger diameter. This is partly because the gas streamlines remain relatively straight as a gas stream approaches a thinner fiber, thereby bringing particles closer to the fiber surface. The particle dendrites formed in early stages of filtration are therefore very efficient in capturing particles because they are of the same diameter as the particles and protrude into the gas stream. In the absence of electrostatic forces, particle dendrites generally increase the collection efficiency as well as the pressure drop across a filter, whereas deposition of liquid droplets has little effect on these two parameters. In contrast, particle loading reduces the collection efficiency of filters made of charged fibers in early stages of filtration since deposited particles diminish electrostatic effects.

Tien et al. [21] proposed a general theory to explain the formation and growth of particle dendrites on a collector placed in an aerosol or hydrosol stream. The theory takes into account the interplay of two intrinsic properties of suspended particles: (1) the finite size of particles and (2) the randomness of the location of individual particles in a fluid stream. The finite size of particles gives rise to two related phenomena: (i) a shadowing effect and (ii) chain deposition. Fig. 2 depicts the forward side of a cross-section of a fiber and three particle trajectories. Particle a follows trajectory $\mathrm{CD}$ and deposits on the fiber at point $\mathrm{A}$. Once particle a has deposited, it creates a shadow area, represented by arc $B_{1} B_{2}$, within which no particle can deposit. Point $B_{1}$ is the point of contact between the fiber and particle $b_{1}$, which follows trajectory $E_{1} F_{1}$ and just moves past particle a when it is at point G. Point $B_{2}$ is the

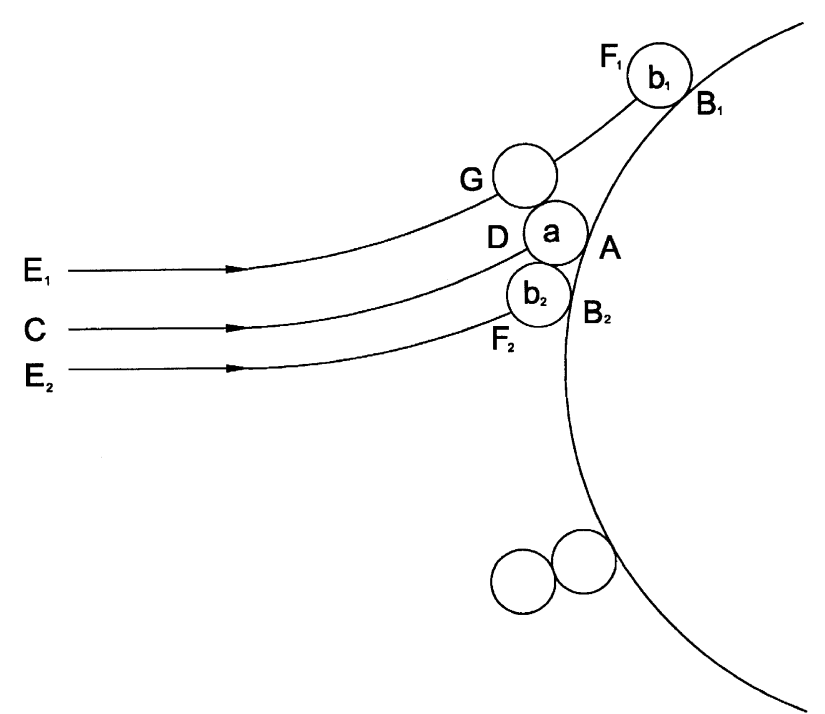

Fig. 2. Illustration of the shadowing effect and chain deposition.

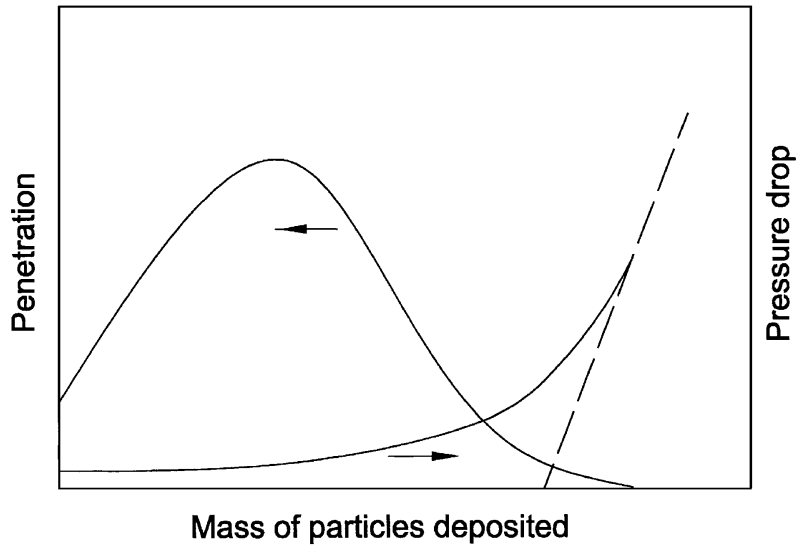

Fig. 3. Penetration and pressure drop curves of an electrically active fibrous filter.

point of contact between the fiber and particle $b_{2}$, which just escapes capture by particle a and deposits on the fiber in its immediate neighborhood. The inhibition of further deposition within the shadow area is an important factor which gives rise to nonuniform deposition. Fig. 2 also depicts a two-particle dendrite formed by the chain deposition of a second particle on a deposited particle.

Numerical simulations based on the theory of Tien et al. are in general agreement with the experimental results obtained with single fibers placed at right angles to an aerosol stream and an externally applied electric field $[22,23]$.

Experimental studies on electret filters [24-27] show that the collection efficiency generally decreases with particle loading in early stages of filtration, but a complex time-dependent behavior exists for different fiber materials. Results of computer simulations [25,28] show the same trends seen in experiments.

Walsh and Stenhouse [29,30] made a study on the loading characteristics of a filter consisting of two types of synthetic fibers of the same diameter $(20 \mu \mathrm{m})$. The fibers were carded together and carried a charge of $5 \mathrm{E}-10 \mathrm{C}$ $\mathrm{m}^{-1}$. The filter had a depth of $0.3 \mathrm{~cm}$ and a packing density of $4 \%$. Fig. 3 illustrates typical penetration and pressure drop curves during the particle loading process in such a filter. A typical penetration curve starts from the initial penetration when the filter is clean, increases to a maximum penetration, and then falls to zero when the deposited particles form a cake. On the other hand, the pressure drop curve rises almost exponentially from the initial value to the point when the filter cake forms. Walsh and Stenhouse defined the clogging point as the point where the asymptote of the pressure drop curve intersects the abscissa. They found that particles which carried higher charges had higher clogging points (higher mass deposited at the clogging point) and, as a consequence, the filtration efficiency degraded more quickly by uncharged particles. Based on these findings, Walsh and Stenhouse suggested that the degradation of filtration efficiency is not a result 
of charge neutralization, but rather a result of charge screening proposed by Brown et al. [31]. The effect of charge screening by deposited particles will diminish as the enhanced collection efficiency of dendrite-shaped particle deposits becomes more significant in the later stages of filtration.

\section{Concluding remarks}

Electrostatic forces have proved to be useful for enhancing the collection efficiency of fibrous filters. Past studies have led to a good understanding of deposition processes by the combined action of electrostatic and mechanical forces in clean filters. However, there is still a marked gap between theory and experiment on the effects of particle loading on collection efficiency. Studies of the flow fields and electric fields around particle dendrites of various lengths are important for the development of a better theory for particle accumulation in fibrous filters. A better understanding of particle loading will facilitate the application of electrostatic forces in aerosol filtration.

\section{References}

[1] C.N. Davies, Air Filtration, Academic Press, London, 1973.

[2] R.C. Brown, Proceedings of the 2nd Word Filtration Congress, London, 1979, pp. 291-301.

[3] R.C. Brown, Air Filtration: An Integrated Approach to the Theory and Applications of Fibrous Filters, Pergamon, Oxford, 1993.

[4] J. Van Turnhout, C. Van Bochove, G.J. Van Veldhuizen, Staub-Reinhalt. Luft 36 (1976) 36-39.

[5] J. Pich, Gas filtration theory, in: M.J. Matteson, C. Orr (Eds.), Filtration-Principles and Practices, Marcel Dekker, New York, 1987, pp. 1-132.

[6] C.S. Wang, Application of electrostatic forces in aerosol filtration-a review, The Third Nisshin Engineering Particle Technology International Seminar-Electrostatic Problem in Powder Technology, Kyoto, Japan, November 29-December 1, 1994, pp. 53-58.

[7] S. Kuwabara, J. Phys. Soc. Jpn. 14 (1959) 527-532.
[8] G. Zebel, J. Colloid Sci. 20 (1965) 522-543.

[9] J. Pich, in: C.N. Davies (Ed.), Aerosol Science, Academic Press, London, 1966.

[10] Z. Wu, J.K. Walters, D.W.P. Thomas, Aerosol Sci. Technol. 30 (1999) 62-70.

[11] R.C. Brown, J. Aerosol Sci. 12 (1981) 349-356.

[12] R.C. Brown, J. Aerosol Sci. 13 (1982) 249-257.

[13] D.O. Banks, G.J. Kurowski, Aerosol Sci. Technol. 12 (1990) 256269.

[14] R. Lathrache, H.J. Fissan, S. Neumann, J. Aerosol Sci. 17 (1986) 446-449.

[15] H.P. Baumgartner, F. Loffler, 2nd International Conference on Electrostatic Precipitation, Kyoto, Japan, 1984.

[16] J. Pich, H. Emi, C. Kanaoka, J. Aerosol Sci. 18 (1987) 29-35.

[17] H. Baumgartner, C. Piesch, H. Umhauer, J. Aerosol Sci. 24 (1993) 945-962.

[18] F.J. Romay, B.Y.H. Liu, S.J. Chae, Aerosol Sci. Technol. 28 (1998) 224-234.

[19] C.E. Billings, Effects of particle accumulation in aerosol filtration, PhD Dissertation, California Institute of Technology, Pasadena, CA, 1966.

[20] C.S. Wang, C.P. Ho, H. Makino, K. Iinoya, AIChE J. 26 (1980) $680-683$

[21] C. Tien, C.S. Wang, D.T. Barot, Science 196 (1977) 983-985.

[22] C.P. Ho, C.S. Wang, Proceedings of International Symposium on Powder Technology, Kyoto, Japan, September 27-October 1, 1981, pp. $565-572$.

[23] C.P. Ho, Collection of solid particles on single cylinders by particle inertia and electrical forces, PhD Dissertation, Syracuse University, Syracuse, NY, 1981.

[24] H. Baumgartner, F. Loffler, H. Umhauer, IEEE Trans. Electr. Insul. 21 (1986) 477-486.

[25] H. Baumgartner, F. Loffler, J. Aerosol Sci. 17 (1986) 438-445.

[26] I. Shimokobe, M. Inoue, J. Aerosol Res., Jpn. 1 (1986) 186-192.

[27] K.B. Tennal, M.K. Mazumder, A. Siag, R.N. Reddy, Part. Sci. Technol. 9 (1991) 19.

[28] C. Kanaoka, Electret Filtration, The Third Nisshin Engineering Particle Technology International Seminar-Electrostatic Problem in Powder Technology, Kyoto, Japan, November 29-December 1, 1994, pp. 59-67.

[29] D.C. Walsh, J.I.T. Stenhouse, J. Aerosol Sci. 28 (1997) 307-321.

[30] D.C. Walsh, J.I.T. Stenhouse, Aerosol Sci. Technol. 29 (1998) 419-432.

[31] R.C. Brown, D. Wake, R. Gray, D.B. Blackford, G.J. Bostock, Ann. Occup. Hyg. 32 (1988) 271-294. 